\title{
Iterative Splitting Methods for Integrodifferential Equations: Theory and Applications
}

\author{
Jürgen Geiser \\ Department of Physics, Ernst-Moritz-Arndt University of Greifswald, Domstraße 14, 17487 Greifswald, Germany \\ Correspondence should be addressed to Jürgen Geiser; geiser@mathematik.hu-berlin.de
}

Received 30 May 2014; Revised 5 August 2014; Accepted 5 August 2014; Published 24 August 2014

Academic Editor: Giuseppe Marino

Copyright (C) 2014 Jürgen Geiser. This is an open access article distributed under the Creative Commons Attribution License, which permits unrestricted use, distribution, and reproduction in any medium, provided the original work is properly cited.

\begin{abstract}
We present novel iterative splitting methods to solve integrodifferential equations. Such integrodifferential equations are applied, for example, in scattering problems of plasma simulations. We concentrate on a linearised integral part and a reformulation to a system of first order differential equations. Such modifications allow for applying standard iterative splitting schemes and for extending the schemes, respecting the integral operator. A numerical analysis is presented of the system of semidiscretised differential equations as abstract Cauchy problems. In the applications, we present benchmark and initial realistic applications to transport problems with scattering terms. We also discuss the benefits of such iterative schemes as fast solver methods.
\end{abstract}

\section{Introduction}

The motivation is to apply fast splitting schemes that have been developed for systems of differential equations, to integrodifferential equations. The applications are related to transport problems with scattering terms, for example, plasma simulations with collision terms; see $[1,2]$. Due to the physics of the situation, the numerical implementation allows decoupling the system into transport and scattering terms, so that the underlying methods can employ optimal splitting schemes.

We concentrate on a semidiscretised transport-scattering equation, given as a Cauchy problem. In the following, we consider the abstract homogeneous Cauchy problem as a system of ordinary integrodifferential equations:

$$
\begin{gathered}
\frac{d \mathbf{u}(t)}{d t}=\mathscr{A}(\mathbf{u}(t))+\int_{0}^{t} \mathscr{B}(\mathbf{u}(s)) d s, \quad t \in[0, T], \\
\mathbf{u}(0)=\mathbf{u}_{0},
\end{gathered}
$$

where $\mathscr{A}, \mathscr{B} \in \mathbf{X} \rightarrow \mathbf{X}$ are bounded and nonlinear operators, which are derived from the semidiscretised transport and scattering operators. $\mathbf{X} \in \mathbb{R}^{n}$ is a Banach space and $\|\cdot\|$ is the corresponding norm in $\mathbf{X}$. The operator $\mathscr{A}$ represents the transport processes, and the operator $\mathscr{B}$ is a given scattering operator.

This paper is organised as follows.
In Section 2 we present the mathematical model and a possible reduced model for further approximations. The numerical schemes are presented in Section 3. The results of some numerical experiments are given in Section 4. The contents of Section 5 contain a summary of the results.

\section{Analytical Approach to the Integrodifferential Equation}

In the following, we discuss the transformation to a system of differential equations in order to apply iterative splitting schemes; see also the decomposition of matrices in [3].

For the linearisation, we make the following assumptions.

Assumption 1. We assume a weak nonlinearity and employ the following simplification.

(1) The Jacobian of $A$, given as $d \mathscr{A} / d \mathbf{u}$, is linearised at $t=$ 0 , which means $\left.(d \mathscr{A} / d \mathbf{u})\right|_{t=0}=A$, where $A \in \mathbf{X} \times \mathbf{X}$.

(2) The operator $\mathscr{B}$ is linearised at $t=0$, which means $\left.B(\mathbf{u})\right|_{t=0}=B$, where $B \in \mathbf{X} \times \mathbf{X}$.

(3) The initial condition of the first derivative is given by $d \mathbf{u}_{0} / d t=A \mathbf{u}_{0}$. 
(4) For the linearised case, the following matrix operations can be applied:

$$
\left(\frac{A A^{t}}{4}+B\right)^{1 / 2}=V\left(\frac{D_{1}}{4}+D_{2}\right) V^{-1},
$$

where $A A^{t}$ and $B$ are simultaneous and diagonalisable, with the same matrix $V$, to the diagonal matrices $D_{1}$ and $D_{2}$. Furthermore, $A A^{t}$ and $B$ have $n$ eigenvectors as a basis for $\mathbf{X} \in \mathbb{R}^{n}$. Further $A A^{t}$ and $B$ commute.

Corollary 2. The integrodifferential equation (1) can be transformed, under Assumption 1, to the following differential equation:

$$
\begin{gathered}
\frac{d^{2} \mathbf{u}(t)}{d^{2} t}=A \frac{d \mathbf{u}(t)}{d t}+B \mathbf{u}(t), \\
\mathbf{u}(0)=\mathbf{u}_{0}, \\
\left.\frac{d \mathbf{u}}{d t}\right|_{t=0}=A \mathbf{u}_{0}
\end{gathered}
$$

where the analytical solution is

$$
\begin{aligned}
\mathbf{u}(t)= & \exp \left(\frac{A}{2} t\right)\left(\cosh (Q t)-\frac{A}{2} Q^{-1} \sinh (Q t)\right) \mathbf{u}_{0}, \\
& +\exp \left(\frac{A}{2} t\right) Q^{-1} \sinh (Q t) A \mathbf{u}_{0}
\end{aligned}
$$

where $Q=(1 / 2) \sqrt{A A^{t} / 4+B}$.

While $A A^{t}$ and $B$ commute, we also have the commutation of $A$ and $Q$ and, for small $t$, we have the solution

$$
\begin{aligned}
\mathbf{u}(t) & =\exp \left(\frac{A}{2} t\right) \cosh (Q t) \mathbf{u}_{0}, \\
& =\frac{1}{2}\left(\exp \left(\left(\frac{A}{2}+Q\right) t\right)+\exp \left(\left(\frac{A}{2}-Q\right) t\right)\right) \mathbf{u}_{0} .
\end{aligned}
$$

Proof. The analytical solution of the second order differential equation (4) is given as follows. We rewrite (4) into

$$
\begin{gathered}
\frac{d \mathbf{u}(t)}{d t}=\mathbf{v}(t), \\
\frac{d \mathbf{v}}{d t}=A \mathbf{v}(t)+B \mathbf{u}(t), \\
\mathbf{u}(0)=\mathbf{u}_{0}, \quad \mathbf{v}(0)=\left.\frac{d \mathbf{u}}{d t}\right|_{t=0}=A \mathbf{u}_{0} .
\end{gathered}
$$

The exponential matrix $\mathscr{A} \in \mathbb{R}^{2 n \times 2 n}$ can be decoupled via the Cayley Hamilton theorem as

$$
\exp (\mathscr{A})=s_{0}(t) \mathscr{I}+s_{1}(t) \mathscr{A}
$$

where $\mathscr{I} \in \mathbb{R}^{2 n \times 2 n}$ is the identity matrix.

The characteristic polynomial of $\mathscr{A}$ is given as

$$
z^{2}-A z-B=\left(z-\lambda_{1}\right)\left(z-\lambda_{2}\right)=0,
$$

and the roots are given as

$$
\lambda_{1,2}=\frac{A}{2} \pm \sqrt{\frac{A A^{t}}{4}+B} .
$$

Then $s_{0}(t)=\exp ((A / 2) t)\left(\cosh (Q t)-(A / 2) Q^{-1} \sinh (Q t)\right)$ and $s_{1}(t)=\exp ((A / 2) t) Q^{-1} \sinh (Q t)$; see also [4]. Therefore the analytical solution is given by (7).

We apply this solution to our integrodifferential equation (1) and, with Assumption 1, the equation is satisfied.

A further simplification can be made to rewrite the integral-differential equation as two first order differential equations. Later such a reduction will allow us to apply fast iterative splitting methods.

Corollary 3. The integrodifferential equation (1) can be transformed, with the help of Assumption 1, to two first order differential equations:

$$
\begin{gathered}
\frac{d \mathbf{u}_{1}(t)}{d t}=\widetilde{A} \mathbf{u}_{1}(t)+\widetilde{B} \mathbf{u}_{1}(t), \\
\mathbf{u}_{1}(0)=\mathbf{u}_{0}, \\
\frac{d \mathbf{u}_{2}(t)}{d t}=\widetilde{A} \mathbf{u}_{2}(t)-\widetilde{B} \mathbf{u}_{2}(t), \\
\mathbf{u}_{2}(0)=\mathbf{u}_{0},
\end{gathered}
$$

where $\widetilde{A}=A / 2$ and $\widetilde{B}=(1 / 2) \sqrt{A A^{t} / 4+B}$.

The analytical solution is

$$
\begin{aligned}
\mathbf{u}(t)= & \frac{1}{2}\left(\mathbf{u}_{1}(t)+\mathbf{u}_{2}(t)\right) \\
= & \frac{1}{2}\left(\exp \left(\left(\frac{A}{2}+\frac{1}{2} \sqrt{\frac{A A^{t}}{4}+B}\right) t\right)\right. \\
& \left.\quad+\exp \left(\left(\frac{A}{2}-\frac{1}{2} \sqrt{\frac{A A^{t}}{4}+B}\right) t\right)\right) \mathbf{u}_{0} .
\end{aligned}
$$

Proof. The analytical solution of the first order differential equations (14) and (16) is obtained using their characteristic polynomials:

$$
\begin{aligned}
& \lambda_{1}-(\widetilde{A}-\widetilde{B})=0, \\
& \lambda_{2}-(\widetilde{A}+\widetilde{B})=0 ;
\end{aligned}
$$

hence the solution is, using the notations of $\widetilde{A}$ and $\widetilde{B}$,

$$
\lambda_{1,2}=\widetilde{A} \pm \widetilde{B}
$$

and therefore the analytical solution is given by (18) with $\widetilde{A}=$ $A / 2, \widetilde{B}=(1 / 2) \sqrt{A A^{t} / 4+B}$.

Therefore this is the solution of our integrodifferential equation (1) under Assumption 1. 


\section{Iterative Splitting Approach to the Integrodifferential Equation}

Operator splitting methods are used to solve complex models in geophysical and environmental physics. They have been developed and applied in [5-7]. Such ideas are the basis for this paper: solving the simpler equations but using higher order discretisation methods for the remaining, more complicated equations. With this aim, we use the operator splitting method and decouple the system of equations as will be described in the following.

We will concentrate on the iterative splitting method. This algorithm is based on an iteration with fixed splitting discretisation step-size $\tau$; namely, on the time interval $\left[t^{n}, t^{n+1}\right]$ we solve the following subproblems consecutively for $i=$ $0,2, \ldots, 2 m$ (cf. $[8,9])$ :

$$
\begin{aligned}
& \frac{\partial c_{i}(t)}{\partial t}=A c_{i}(t)+B \int_{0}^{t} c_{i-1}(s) d s, \\
& \quad \text { with } c_{i}\left(t^{n}\right)=c^{n}, \quad c_{0}\left(t^{n}\right)=c^{n}, \quad c_{-1}=0.0, \\
& \frac{\partial c_{i+1}(t)}{\partial t}=A c_{i}(t)+B \int_{0}^{t} c_{i+1}(s) d s, \\
& \quad \text { with } c_{i+1}\left(t^{n}\right)=c^{n}
\end{aligned}
$$

where $c^{n}$ is the known split approximation at the time level $t=$ $t^{n}$. The split approximation at the time level $t=t^{n+1}$ is defined by $c^{n+1}=c_{2 m+1}\left(t^{n+1}\right)$. (Clearly, the function $c_{i+1}(t)$ depends on the interval $\left[t^{n}, t^{n+1}\right]$, too, but, for the sake of simplicity, in our notation, we omit the dependence on $n$.)

For the simplification, we apply two standard iterative splitting schemes to the first order differential equations.

The first iterative process is

$$
\begin{aligned}
& \frac{\partial c_{1, i}(t)}{\partial t}=\widetilde{A} c_{1, i}(t)+\widetilde{B} c_{1, i-1}(t), \\
& \quad \text { with } c_{1, i}\left(t^{n}\right)=c^{n}, \quad c_{1,0}\left(t^{n}\right)=c^{n}, \quad c_{1,-1}=0.0 \\
& \frac{\partial c_{1, i+1}(t)}{\partial t}=\widetilde{A} c_{1, i}(t)+\widetilde{B} c_{1, i+1}(t), \\
& \text { with } c_{1, i+1}\left(t^{n}\right)=c^{n}
\end{aligned}
$$

and the second iterative process is

$$
\begin{aligned}
& \frac{\partial c_{2, i}(t)}{\partial t}=\widetilde{A} c_{2, i}(t)-\widetilde{B} c_{2, i-1}(t), \\
& \quad \text { with } c_{2, i}\left(t^{n}\right)=c^{n}, \quad c_{2,0}\left(t^{n}\right)=c^{n}, \quad c_{1,-1}=0.0, \\
& \frac{\partial c_{2, i+1}(t)}{\partial t}=\widetilde{A} c_{2, i}(t)-\widetilde{B} c_{2, i+1}(t), \\
& \quad \text { with } c_{2, i+1}\left(t^{n}\right)=c^{n},
\end{aligned}
$$

where $c^{n}$ is the known split approximation at the time level $t=t^{n}$. The split approximation at the time level $t=t^{n+1}$ is defined by $c^{n+1}=(1 / 2)\left(c_{1,2 m+1}\left(t^{n+1}\right)+c_{2,2 m+1}\left(t^{n+1}\right)\right)$.

In the following, we will analyse the convergence and the rate of convergence of the methods (22) and (23) for $m$ tending to infinity for the linear operators $\widetilde{A}, \widetilde{B}: \mathbf{X} \rightarrow \mathbf{X}$ using Assumption 1.

It suffices to discuss one case, since the other case is the same with only a change in the sign of the operator $\widetilde{B}$.

Theorem 4. Let us consider the abstract Cauchy problem in a Banach space $\mathbf{X}$ :

$$
\begin{gathered}
\partial_{t} c(t)=\widetilde{A} c(t)+\widetilde{B} c(t), \quad 0<t \leq T, \\
c(0)=c_{0},
\end{gathered}
$$

where $\widetilde{A}, \widetilde{B}, \widetilde{A}+\widetilde{B}: \mathbf{X} \rightarrow \mathbf{X}$ are given linear operators which are generators of the $C_{0}$-semigroup and $c_{0} \in \mathbf{X}$ is a given element. Then the iteration process (22) is convergent and the rate of convergence is of second order.

Proof. The proof is given in [10].

In the following Algorithm 5, we have combined the first and second iterative processes.

Algorithm 5. We apply $\left(-\widetilde{B}^{i}\right)=(-1)^{i} \widetilde{B}$ and $\exp (\widetilde{B} t)$ $\exp (-\widetilde{B} t)=I$. The time-steps are $t^{j}, j=0, \ldots, N$, with $t^{j+1}-t^{j}=\Delta t$, and where $I \in \mathbb{N}^{+}$is a given number of iterative steps.

We have the following algorithm:

$$
\begin{aligned}
& \partial c_{1 / 2}^{i}(t) / \partial t=\widetilde{A} c_{1 / 2}^{i}(t) \pm \widetilde{B} c^{i-1}(t), \text { with } c_{1 / 2}^{i}\left(t^{n}\right)= \\
& c_{1 / 2}^{i-1}\left(t^{n+1}\right),
\end{aligned}
$$

the starting values $c_{1 / 2}^{0}\left(t^{n}\right)=c\left(t^{n}\right)$,

results of the last iteration, $c_{1 / 2}^{-1}\left(t^{n}\right)=0.0$,

$\partial c_{1 / 2}^{i+1}(t) / \partial t=\widetilde{A} c_{1 / 2}^{i}(t) \pm \widetilde{B} c_{1 / 2}^{i+1}(t)$,

with $c_{1 / 2}^{i+1}\left(t^{n}\right)=c_{1 / 2}^{i}\left(t^{n+1}\right)$,

if $\epsilon>\max _{j=1,2}\left|c_{j}^{i+1}\left(t^{n+1}\right)-c_{j}^{i-1}\left(t^{n+1}\right)\right|$ or $i+1=$ $I$ we stop

$c\left(t^{n+1}\right)=(1 / 2)\left(c_{1}^{i+1}\left(t^{n+1}\right)+c_{2}^{i+1}\left(t^{n+1}\right)\right)$

we go to the next time-step $n=n+1$ till $n+1=N$, else $i=i+1$

\section{Numerical Experiments}

For the applications part, we will now treat test problems and real-life applications to transport-scattering problems.

The first examples are to validate our novel splitting and solver methods; then, in the subsequent examples, we use more delicate coupled differential equations.

4.1. First Example: A Matrix Problem with Integral Term. We deal with simpler integrodifferential equations:

$$
c^{\prime}(t)=c+\int_{0}^{t} c(t) d t, \quad t \in[0,1]
$$


where we take $\int_{0}^{t} c(t) d t=t c(t)$ as a first order approximation of the integral and treat

$$
\begin{gathered}
c^{\prime}(t)=c+t c(t), \quad t \in[0, T], \\
c(0)=c\left(t_{0}\right)=1,
\end{gathered}
$$

where $T=10.0$ and we have the analytical solution for the approximation

$$
c(t)=\exp \left(t+\frac{t^{2}}{2}\right) u(0) .
$$

We split this into

$$
\begin{gathered}
A=1, \\
B(t)=t .
\end{gathered}
$$

We have the following solutions for the iterative scheme:

$$
\begin{aligned}
& c_{1}(t)= \exp \left(A\left(t^{n+1}-t\right)\right) c\left(t^{n}\right), \quad t \in\left(t^{n}, t^{n+1}\right], \\
& c_{2}(t)= \exp \left(\int_{t_{n}}^{t^{n+1}} B(t) d t\right) c\left(t^{n}\right) \\
&+\int_{t^{n}}^{t^{n+1}} \exp \left(\int_{s}^{t^{n+1}} B\left(t^{n+1}-t\right)\right) d t A c_{1}(s) d s \\
& t \in\left(t^{n}, t^{n+1}\right],
\end{aligned}
$$

where $n=0,1, \ldots, N$ and $t^{N}=T$ while the time-steps are given by $\Delta t=t^{n+1}-t^{n}$.

We employ the following recurrence relations, distinguishing between even and odd iterations.

For the odd iterations, $i=2 m+1$, for $m=0,1,2, \ldots$,

$$
\begin{aligned}
c_{i}(t)= & \exp \left(A\left(t-t^{n}\right)\right) c\left(t^{n}\right) \\
& +\int_{t^{n}}^{t} \exp (s A) B(s) c_{i-1}\left(t^{n+1}-s\right) d s, \\
& t \in\left(t^{n}, t^{n+1}\right] .
\end{aligned}
$$

For the even iterations, $i=2 m$, for $m=1,2, \ldots$,

$$
\begin{aligned}
& c_{i}(t)= \exp \left(\int_{t_{n}}^{t^{n+1}} B(s) d s\right) c\left(t^{n}\right) \\
&+\int_{t^{n}}^{t} \exp \left(\int_{0}^{s} B(t) d t\right) A c_{i-1}\left(t^{n+1}-s\right) d s, \\
& \quad t \in\left(t^{n}, t^{n+1}\right] .
\end{aligned}
$$

We have a blow-up solution of exponential character in long time behavior; see Figure 1. Therefore, it is advisable that the integrator should be highly accurate for long time behavior.

Table 1 gives the numerical results of the iterative splitting scheme.

Figure 2 presents the one-sided and two-sided iterative results.
Remark 6. In the experiments, we obtain improved results with each additional step. By the way, the solution blows up and we have to use also very fine time-steps to control the errors. Optimal results are obtained by using the integral part (stiff part) as the implicit part in the iteration (one-sided over $B)$.

4.2. Second Example: Integrodifferential Equation. We treat the system of integrodifferential equations

$$
\mathbf{c}^{\prime}(t)=A \mathbf{c}+B \int_{0}^{t} \mathbf{c}(t) d t, \quad t \in[0,1]
$$

where we have the analytical solution

$$
\begin{gathered}
\mathbf{c}(t)=\exp \left(A t+\frac{1}{2} B t^{2}\right) \mathbf{c}(0), \quad t \in[0, T], \\
\mathbf{c}(0)=\mathbf{c}\left(t_{0}\right)=(1, \ldots, 1)^{t},
\end{gathered}
$$

where $A, B \in \mathrm{IR}^{m \times m}$ and, for example, we take $m=10$.

We have employed the integral approximation

$$
\begin{gathered}
\mathbf{c}^{\prime}(t)=A \mathbf{c}+B \int_{0}^{t} \mathbf{c}(t) d t \\
\mathbf{c}^{\prime}(t)=A \mathbf{c}+B t \mathbf{c}(t) .
\end{gathered}
$$

The matrices are given by

$$
\begin{gathered}
A=\Lambda_{1}+\Lambda_{2}=\left(\begin{array}{cccc}
-\lambda_{1} & 0 & \ldots & 0 \\
0 & -\lambda_{1} & 0 & \vdots \\
\vdots & \ddots & \ddots & \ddots \\
0 & \ldots & 0 & -\lambda_{1}
\end{array}\right) \\
+\left(\begin{array}{cccc}
0 & 0 & \ldots & 0 \\
\lambda_{2} & 0 & 0 & \vdots \\
\vdots & \ddots & \ddots & \ddots \\
0 & \ldots & \lambda_{2} & 0
\end{array}\right), \\
B=\left(\begin{array}{cccc}
-\lambda_{3} & 0 & \ldots & 0 \\
0 & -\lambda_{3} & 0 & \vdots \\
\vdots & \ddots & \ddots & \ddots \\
0 & \ldots & 0 & -\lambda_{3}
\end{array}\right)
\end{gathered}
$$

where the parameters are $\lambda_{1}=0.02, \lambda_{2}=0.02$, and $\lambda_{3}=$ 0.01 .

The splitting into the two operators $A$ and $B$ is made in order to accelerate the computation of the exponential matrices $\exp (A)$ and $\exp (B)$. 


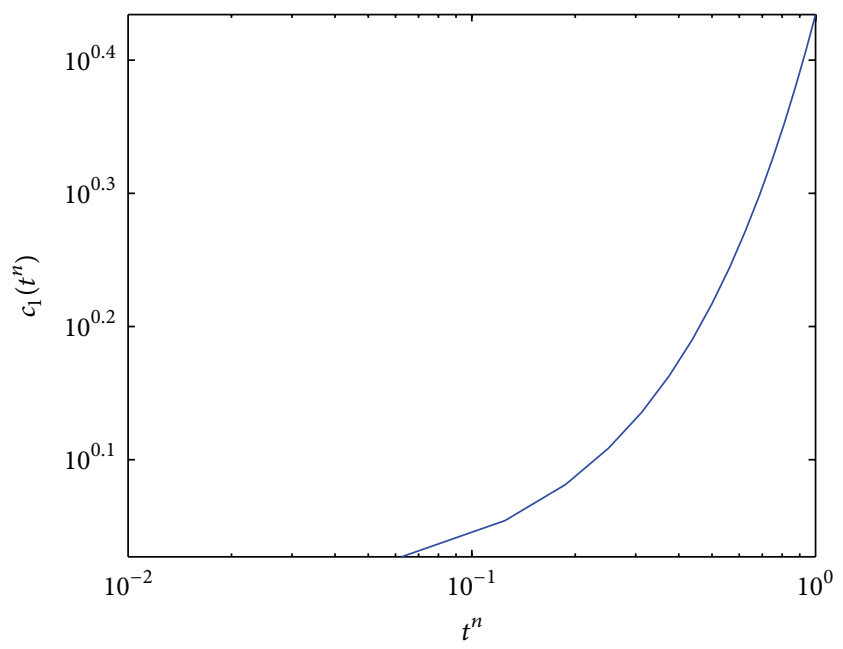

(a)

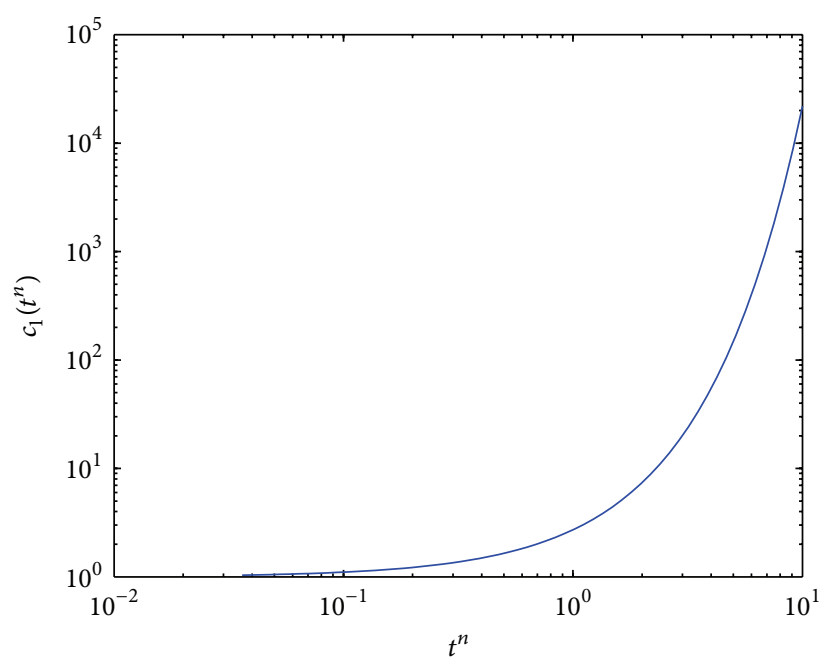

(b)

FIGURE 1: Short time and long time behavior of the solution ((a) $T=1$, (b) $T=10)$.

TABLE 1: Numerical experiment with 10 iterative steps for the first example.

\begin{tabular}{lccccc}
\hline & $\Delta t=1$ & $\Delta t=0.5$ & $\Delta t=0.25$ & $\Delta t=2^{-3}$ & $\Delta t=2^{-4}$ \\
\hline$c_{1}$ & 1.7634 & 0.4958 & 0.1793 & 0.0753 & 0.0343 \\
$c_{2}$ & 0.8628 & 0.1444 & 0.0282 & 0.0061 & 0.0014 \\
$c_{3}$ & 0.2220 & 0.0104 & $5.2127 e-04$ & $2.8455 e-05$ & $1.6511 e-06$ \\
$c_{4}$ & 0.1116 & 0.0041 & $1.8660 e-04$ & $9.7846 e-06$ & $5.5769 e-07$ \\
$c_{5}$ & 0.0971 & 0.0039 & $1.8367 e-04$ & $9.7418 e-06$ & $5.5644 e-07$ \\
$c_{6}$ & 0.0956 & 0.0039 & $1.8365 e-04$ & $9.7418 e-06$ & $5.5644 e-07$ \\
$c_{7}$ & 0.0954 & 0.0039 & $1.8365 e-04$ & $9.7416 e-06$ & $5.5556 e-07$ \\
$c_{8}$ & 0.0954 & 0.0039 & $1.8366 e-04$ & $9.7343 e-06$ & 07 \\
\hline
\end{tabular}

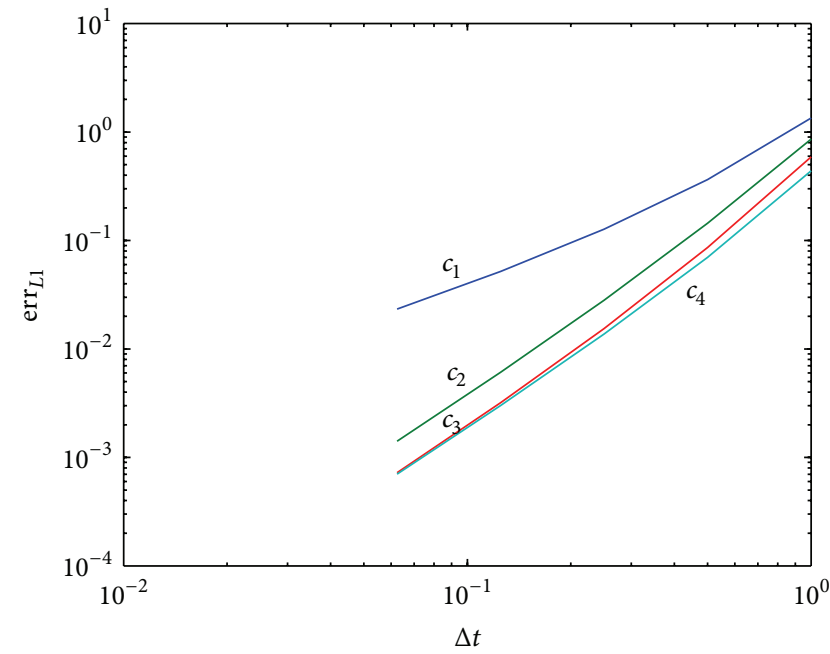

(a)

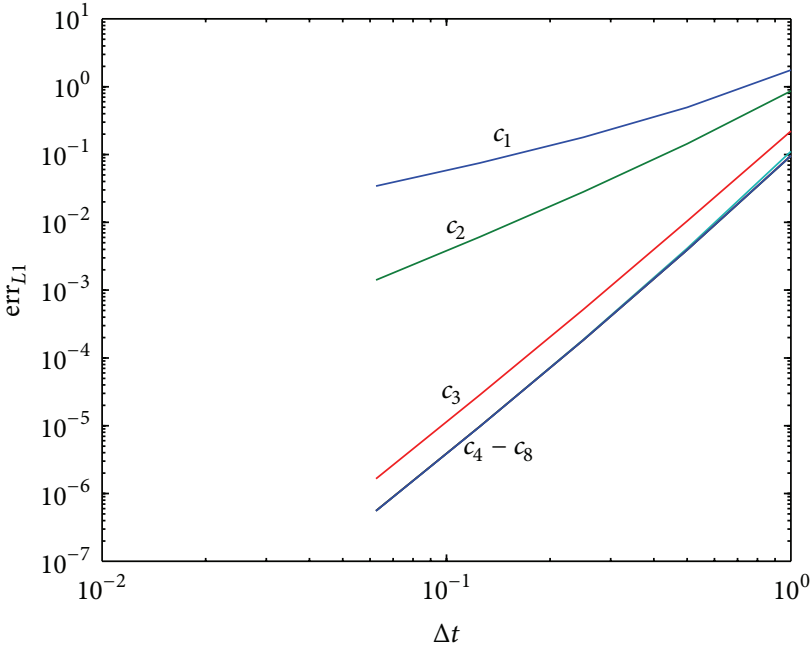

(b)

Figure 2: Numerical errors of the one-sided and two-sided splitting schemes: one-sided splitting over $A$ (a) and two-sided splitting scheme alternating between $A$ and $B$ (b) with $1, \ldots, 8$ iterative steps. 


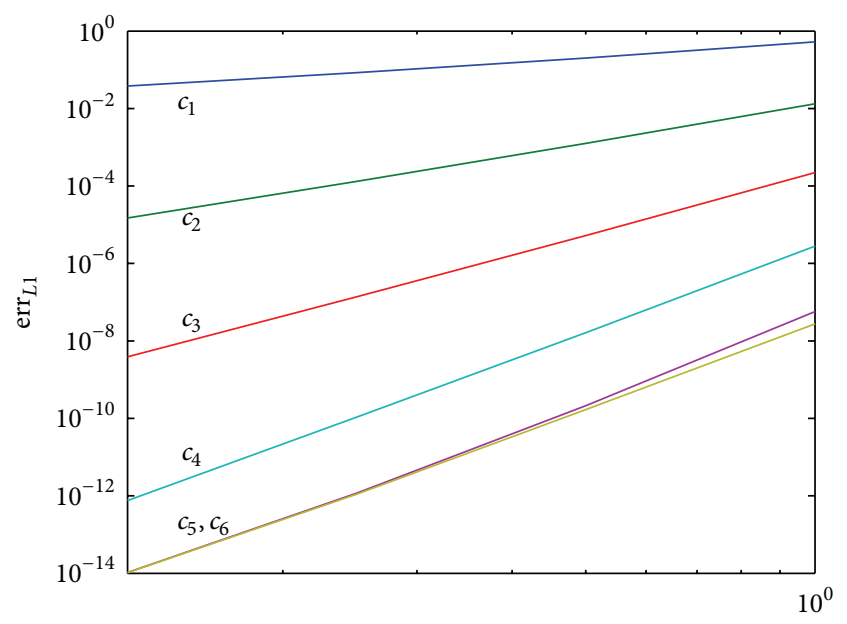

$\Delta t$

(a)

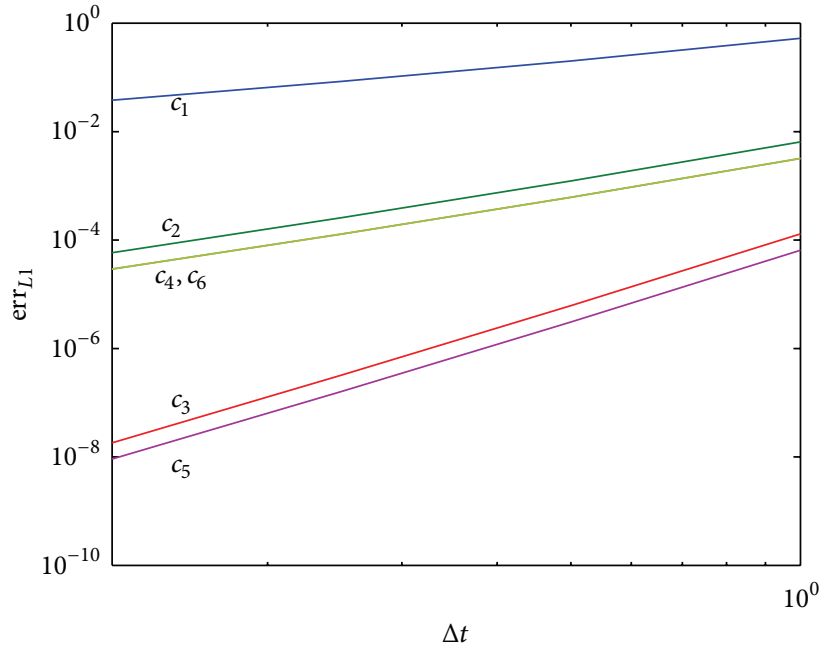

(b)

FIGURE 3: Numerical errors of the one-sided and two-sided splitting schemes: one-sided splitting over $A$ (a) and two-sided splitting scheme alternating between $A$ and $B$ (b) with $1, \ldots, 6$ iterative steps.

(1) The operator $A$ is computed as

$$
\begin{gathered}
\exp (A t)=\exp \left(\Lambda_{1} t\right) \exp \left(\Lambda_{2} t\right) \\
\exp \left(\Lambda_{1} t\right)=\left(\begin{array}{cccc}
\exp \left(-\lambda_{1} t\right) & 0 & \cdots & 0 \\
0 & \exp \left(-\lambda_{1} t\right) & 0 & \vdots \\
\vdots & \ddots & \ddots & \vdots \\
0 & \cdots & 0 & \exp \left(-\lambda_{1} t\right)
\end{array}\right), \\
\exp \left(\Lambda_{2} t\right)=I+\Lambda_{2} t+\frac{1}{2} \Lambda_{2}^{2} t^{2}+\frac{1}{6} \Lambda_{2}^{3} t^{3} \\
+\cdots+\frac{1}{(q-1) !} \Lambda_{2}^{q-1} t^{q-1}
\end{gathered}
$$

where $\Lambda_{2}^{q}=0$.

We have the following solutions for the iterative scheme:

$$
\begin{aligned}
c_{1}(t)=\exp \left(A\left(t^{n+1}-t\right)\right) c\left(t^{n}\right), \quad t \in\left(t^{n}, t^{n+1}\right] \\
c_{2}(t)=\exp \left(B \frac{\left(t^{n+1}-t\right)^{2}}{2}\right) c\left(t^{n}\right) \\
+\int_{t^{n}}^{t^{n+1}} \exp \left(B \frac{(t-s)^{2}}{2}\right) A c_{1}(s) d s \\
t \in\left(t^{n}, t^{n+1}\right]
\end{aligned}
$$

where $n=0,1, \ldots, N$ and $t^{N}=T$ while the time-steps are given by $\Delta t=t^{n+1}-t^{n}$.

We employ the following recurrence relations, distinguishing between even and odd iterations.
For the odd iterations, $i=2 m+1$, for $m=0,1,2, \ldots$,

$$
\begin{aligned}
c_{i}(t)= & \exp \left(A\left(t-t^{n}\right)\right) c\left(t^{n}\right) \\
& +\int_{t^{n}}^{t} \exp ((t-s) A) B s c_{i-1}(s) d s, \\
& t \in\left(t^{n}, t^{n+1}\right] .
\end{aligned}
$$

For the even iterations, $i=2 m$, for $m=1,2, \ldots$,

$$
\begin{aligned}
c_{i}(t)= & \exp \left(B \frac{\left(t^{n+1}-t\right)^{2}}{2}\right) c\left(t^{n}\right) \\
& +\int_{t^{n}}^{t} \exp \left(B \frac{(t-s)^{2}}{2}\right) A c_{i-1}(s) d s, \\
& t \in\left(t^{n}, t^{n+1}\right] .
\end{aligned}
$$

For the integral computations, we apply higher order schemes, the Newton-Cotes formula (e.g., Simpson's rule and Boole's rule); see [11].

We acchieve for the second example (vectorial benchmark problem) the same accuracy for its first component as in the first example (scalar benchmark problem).

Figure 3 presents the one-sided and two-sided iterative results.

The computational results are given in Figure 4, which presents the one-sided and two-sided iterative results.

Remark 7. In the experiments, we obtain improved results with each additional step. By the way, the solution blows up and so we have to use very fine time-steps to control the errors. The optimal results are obtained by using the integral part (stiff part) as the implicit part in the iteration (one-sided over $B$ ). 


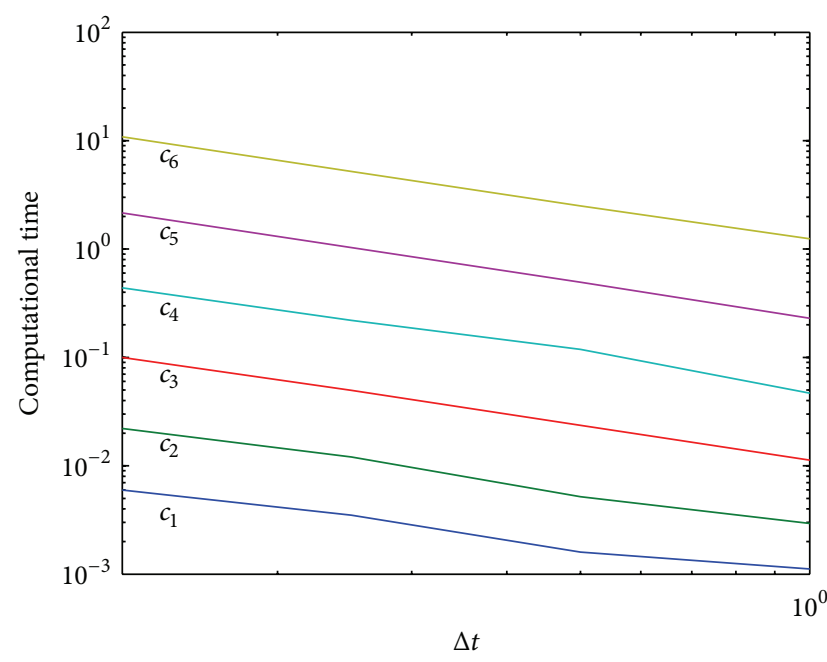

(a)

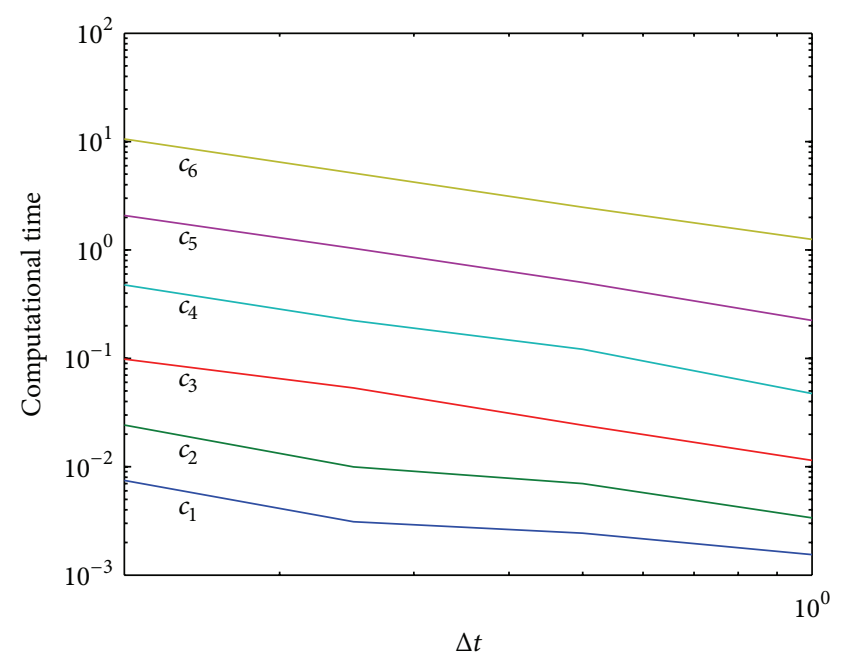

(b)

Figure 4: The computational time of the one-sided and two-sided splitting schemes: one-sided splitting over $A$ (a) and two-sided splitting scheme alternating between $A$ and $B$ (b) with $1, \ldots, 6$ iterative steps.

4.2.1. Exact Integral Part Based on the Splitting Approach. We treat a system of integrodifferential equations

$$
\mathbf{c}^{\prime}(t)=A \mathbf{c}+B \int_{0}^{t} \mathbf{c}(t) d t, \quad t \in[0,1]
$$

where we assume that the primitive of $\mathbf{c}$ is $\mathbf{C}(0)=\mathbf{0}$.

We have the analytical solution

$$
\begin{aligned}
\mathbf{u}(t)=\frac{1}{2}( & \exp \left(\left(\frac{A}{2}+\sqrt{\frac{A A^{t}}{4}+B}\right) t\right) \\
& \left.+\exp \left(\left(\frac{A}{2}-\sqrt{\frac{A A^{t}}{4}+B}\right) t\right)\right) \mathbf{u}_{0}
\end{aligned}
$$

where $\widetilde{A}=A / 2, \widetilde{B}=\sqrt{A A^{t} / 4+B} \in \mathbb{R}^{m \times m}$ and, for example, we take $m=10$.

The treatment of the square root for matrices is given in $[12,13]$.

We have the following matrices:

$$
\begin{aligned}
A=\Lambda_{1}+\Lambda_{2}= & \left(\begin{array}{cccc}
-\lambda_{1} & 0 & \ldots & 0 \\
0 & -\lambda_{1} & 0 & \vdots \\
\vdots & \ddots & \ddots & \ddots \\
0 & \ldots & 0 & -\lambda_{1}
\end{array}\right) \\
& +\left(\begin{array}{cccc}
0 & 0 & \ldots & 0 \\
\lambda_{2} & 0 & 0 & \vdots \\
\vdots & \ddots & \ddots & \ddots \\
0 & \ldots & \lambda_{2} & 0
\end{array}\right)
\end{aligned}
$$

$$
B=\left(\begin{array}{cccc}
-\lambda_{3} & 0 & \ldots & 0 \\
0 & -\lambda_{3} & 0 & \vdots \\
\vdots & \ddots & \ddots & \ddots \\
0 & \ldots & 0 & -\lambda_{3}
\end{array}\right)
$$

where the parameters are $\lambda_{1}=0.02, \lambda_{2}=0.02$, and $\lambda_{3}=0.01$.

The exponentials of these operators are computed using Padé approximants; see the discussion in [11].

We have the following solutions for the two iterative schemes.

Algorithm 8. Iterative process for $c_{1}$ :

$$
\begin{array}{r}
c_{1,1}(t)=\exp \left(\widetilde{A}\left(t^{n+1}-t\right)\right) c\left(t^{n}\right), \quad t \in\left(t^{n}, t^{n+1}\right], \\
c_{1,2}(t)=\exp \left(\widetilde{B}\left(t^{n+1}-t\right)\right) c\left(t^{n}\right) \\
+\int_{t^{n}}^{t^{n+1}} \exp \left(\left(t^{n+1}-s\right) \widetilde{B}\right) \widetilde{A} c_{1,1}(s) d s, \\
t \in\left(t^{n}, t^{n+1}\right] .
\end{array}
$$

For the odd iterations, $i=2 m+1$, we have, for $m=0,1,2, \ldots$,

$$
\begin{aligned}
& c_{1, i}(t)= \exp \left(\widetilde{A}\left(t-t^{n}\right)\right) c\left(t^{n}\right) \\
&+\int_{t^{n}}^{t} \exp ((t-s) \widetilde{A}) \widetilde{B} c_{1, i-1}(s) d s, \\
& \quad t \in\left(t^{n}, t^{n+1}\right] .
\end{aligned}
$$


TABLE 2: Numerical experiment with three iterative steps for the second example and the first component.

\begin{tabular}{cccccc}
\hline & $\Delta t=1$ & $\Delta t=0.5$ & $\Delta t=0.25$ & $\Delta t=2^{-3}$ & $\Delta t=2^{-4}$ \\
\hline$c_{1}$ & 1.7634 & 0.4958 & 0.1793 & 0.0753 & 0.0343 \\
$c_{2}$ & 0.8628 & 0.1444 & 0.0282 & 0.0061 & 0.0014 \\
$c_{3}$ & 0.2220 & 0.0104 & $5.2127 e-04$ & $2.8455 e-05$ & $1.6511 e-06$ \\
\hline
\end{tabular}

For the even iterations, $i=2 m$, we have, for $m=1,2, \ldots$,

$$
\begin{aligned}
c_{1, i}(t)= & \exp \left(\widetilde{B}\left(t-t^{n}\right)\right) c\left(t^{n}\right) \\
& +\int_{t^{n}}^{t} \exp (\widetilde{B}(t-s)) \widetilde{A} c_{1, i-1}(s) d s, \\
& t \in\left(t^{n}, t^{n+1}\right],
\end{aligned}
$$

where $n=0,1, \ldots, N, t^{N}=T$, and the time-steps are given by $\Delta t=t^{n+1}-t^{n}$.

Algorithm 9. Iterative process for $c_{2}$ :

$$
\begin{array}{rl}
c_{2,1}(t)= & \exp \left(\widetilde{A}\left(t^{n+1}-t\right)\right) c\left(t^{n}\right), \quad t \in\left(t^{n}, t^{n+1}\right], \\
c_{2,2}(t)= & \exp \left(-\widetilde{B}\left(t^{n+1}-t\right)\right) c\left(t^{n}\right) \\
& +\int_{t^{n}}^{t^{n+1}} \exp \left(-\left(t^{n+1}-s\right) \widetilde{B}\right) \widetilde{A} c_{1,1}(s) d s \\
t & t\left(t^{n}, t^{n+1}\right] .
\end{array}
$$

For the odd iterations, $i=2 m+1$, we have, for $m=0,1,2, \ldots$,

$$
\begin{aligned}
& c_{2, i}(t)= \exp \left(\widetilde{A}\left(t-t^{n}\right)\right) c\left(t^{n}\right) \\
&+\int_{t^{n}}^{t} \exp ((t-s) \widetilde{A})(-\widetilde{B}) c_{2, i-1}(s) d s, \\
& \quad t \in\left(t^{n}, t^{n+1}\right] .
\end{aligned}
$$

For the even iterations, $i=2 m$, we have, for $m=1,2, \ldots$,

$$
\begin{aligned}
& c_{2, i}(t)= \exp \left(-\widetilde{B}\left(t-t^{n}\right)\right) c\left(t^{n}\right) \\
&+\int_{t^{n}}^{t} \exp (-\widetilde{B}(t-s)) \widetilde{A} c_{2, i-1}(s) d s, \\
& t \in\left(t^{n}, t^{n+1}\right],
\end{aligned}
$$

where $n=0,1, \ldots, N, t^{N}=T$, and the time-steps are given by $\Delta t=t^{n+1}-t^{n}$.

The solution after each iterative step with the two single solutions is

$$
c_{i}(t)=\frac{1}{2}\left(c_{1, i}(t)+c_{2, i}(t)\right) .
$$

For the integral computations, we apply higher order schemes, Newton-Cotes formulas (e.g., Simpson's rule and Boole's rule); see [11].
TABLE 3: The computational time of all three experiments.

\begin{tabular}{lc}
\hline Algorithm & Computational time in sec \\
\hline num. approach-twoSide & 24.3976 \\
num. approach-oneSideA & 24.7610 \\
num. approach-oneSideB & 24.7252 \\
\hline exact approach-twoSide & 67.0159 \\
exact approach-oneSideA & 68.4638 \\
exact approach-oneSideB & 69.1291 \\
\hline exact optimised approach-twoSide & 36.3361 \\
exact optimised approach-oneSideA & 36.9736 \\
exact optimised approach-oneSideB & 35.8903 \\
\hline
\end{tabular}

Table 2 shows the numerical results of the iterative splitting scheme. We also acchieve similar results for the first component of the vectorial benchmark problem as in its scalar form in the first experiment.

Figure 5 presents the one-sided and two-sided iterative results for an optimised approach.

The computational results are given in Figure 6; we present the one-sided and two-sided iterative results for an optimised approach.

Remark 10. In the experiments, we obtain improved results with each additional step. By the way, the solution blows up and we have to use very fine time-steps to control the errors. The optimal results are obtained by using the integral part (stiff part) as the implicit part in the iteration (one-sided over $B)$.

Remark 11. To optimise the algorithms, we combine both of the iterative cycles with the idea of $-B^{i}=(-1)^{i} B$ and in the Padé approximants we apply $\exp (B t) \exp (-B t)=I$; see Algorithm 5.

Remark 12. Table 3 presents the computational times of the three experiments. While the exact approach based on the splitting idea of higher order differential equations obtains more accurate solutions, its drawback is its higher computational costs. We reduce such costs by using the symmetry of the underlying iterative schemes and reach nearly the same computational time as in the numerical approach scheme.

4.3. Real-Life Example: One-Phase Problem. The next example is a simplified real-life problem with a neutron transport equation, which includes the gain and loss of a neutron.

We concentrate on the computational benefits of a fast computation of the iterative scheme, given with matrix exponential functions. 


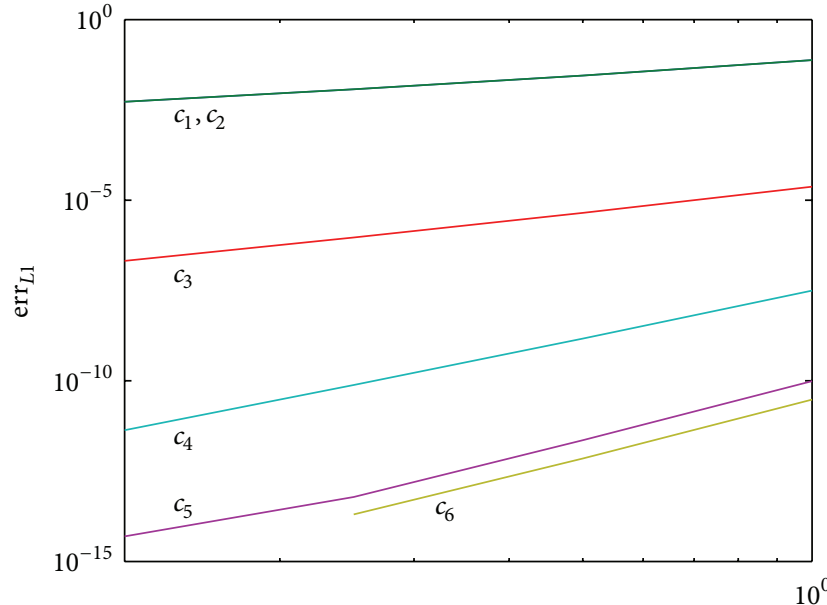

$\Delta t$

(a)

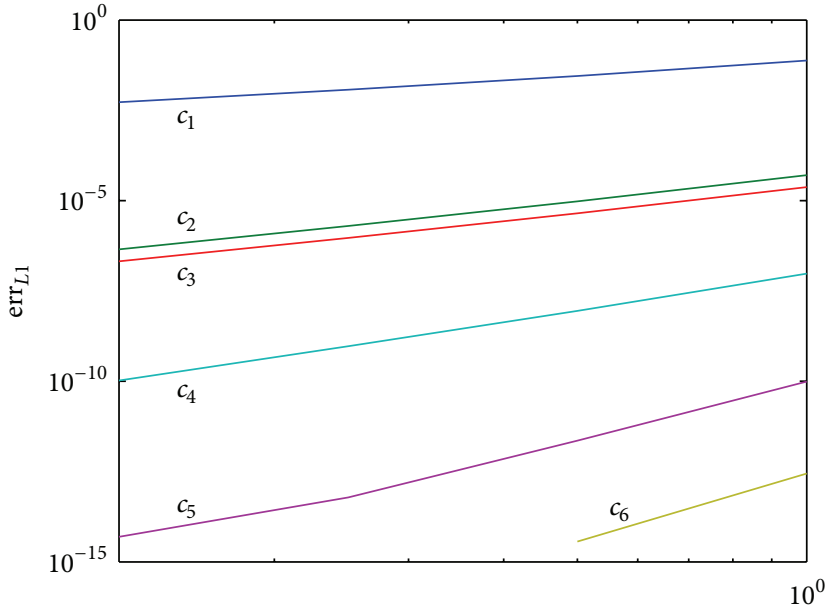

$\Delta t$

(b)

Figure 5: Numerical errors of the one-sided and two-sided splitting schemes for an optimised approach: one-sided splitting over $A$ (a) and two-sided splitting scheme alternating between $A$ and $B(b)$ with $1, \ldots, 6$ iterative steps.

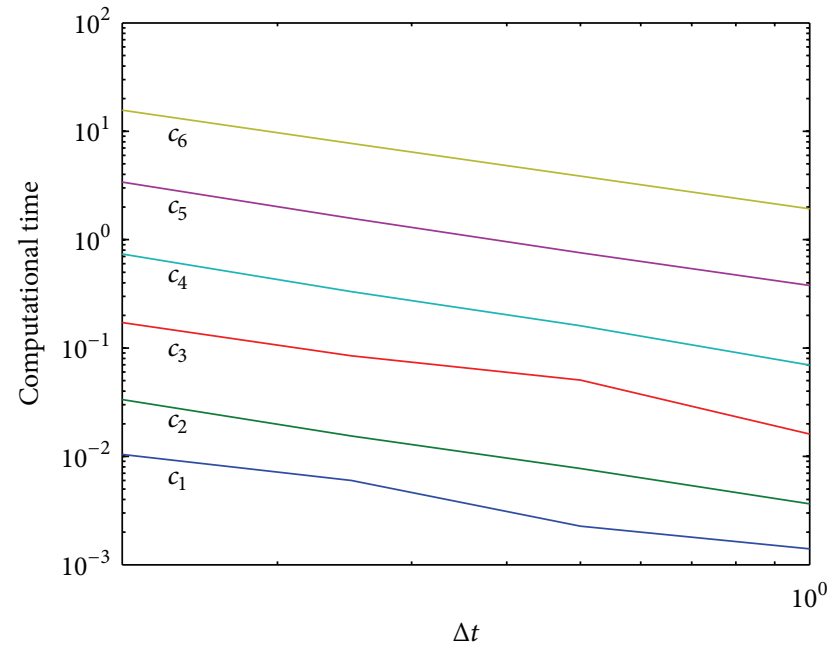

(a)

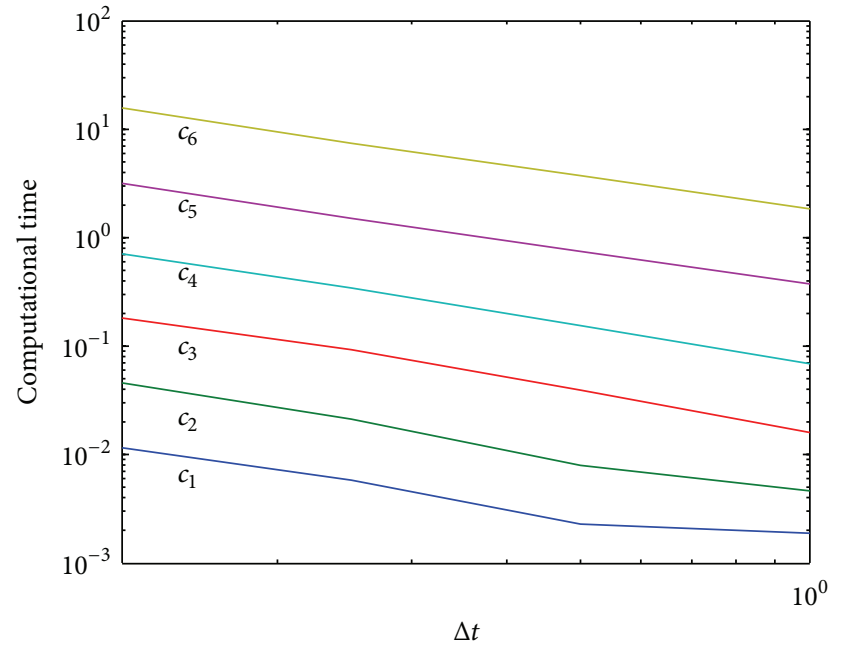

(b)

FIGURE 6: The computational time of the one-sided and two-sided splitting schemes for an optimised approach: one-sided splitting over $A$ (a) and two-sided splitting scheme alternating between $A$ and $B$ (b) with $1, \ldots, 6$ iterative steps.

The equation is

$$
\begin{gathered}
\partial_{t} c+\nabla \cdot \mathbf{F} c=-\lambda_{1} c+\int_{0}^{t} \lambda_{2} c(x, t) d t, \quad \text { in } \Omega \times[0, t], \\
\mathbf{F}=\mathbf{v}-D \nabla, \\
c(\mathbf{x}, t)=c_{0}(\mathbf{x}), \quad \text { on } \Omega, \\
c(\mathbf{x}, t)=c_{1}(\mathbf{x}, t), \quad \text { on } \partial \Omega \times[0, t] .
\end{gathered}
$$

In the following, we deal with the semidiscretised equation given by the matrices

$$
\partial_{t} \mathbf{C}=\left(A-\Lambda_{1}\right) \mathbf{C}+\int_{0}^{t} \Lambda_{2} \mathbf{C}(s) d s
$$

where $\mathbf{C}=\left(c_{1}, \ldots, c_{m}\right)^{T}$ is the solution of the species in the mobile phase in each spatial discretisation point $(i=$ $1, \ldots, m)$.

We have the following two operators for the splitting method:

$$
\begin{aligned}
A & =\frac{D}{\Delta x^{2}} \cdot\left(\begin{array}{ccccc}
-2 & 1 & & & \\
1 & -2 & 1 & & \\
& \ddots & \ddots & \ddots & \\
& & 1 & -2 & 1 \\
& & & 1 & -2
\end{array}\right) \\
& +\frac{v}{\Delta x} \cdot\left(\begin{array}{ccccc}
1 & & & & \\
-1 & 1 & & & \\
& \ddots & \ddots & & \\
& & -1 & 1 & \\
& & & -1 & 1
\end{array}\right) \in \mathbb{R}^{m \times m}
\end{aligned}
$$




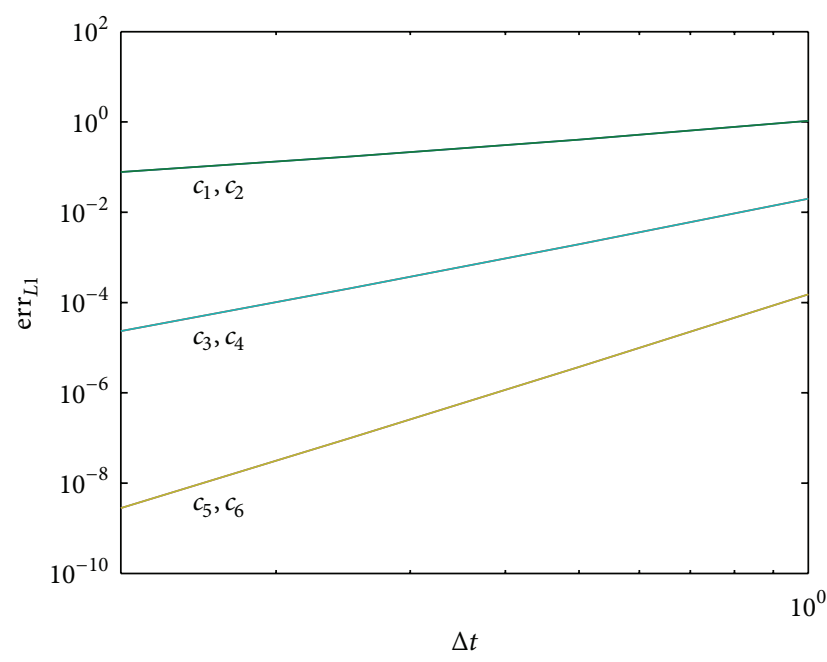

(a)

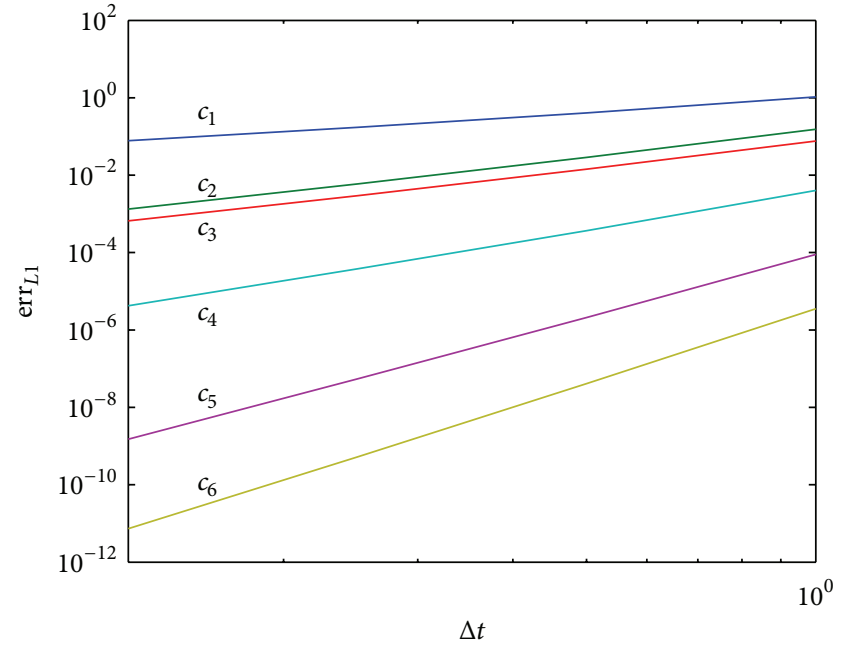

(b)

Figure 7: Numerical errors of the one-sided and two-sided splitting schemes: one-sided splitting over $A$ (a) and two-sided splitting scheme alternating between $A$ and $B$ (b) with $1, \ldots, 6$ iterative steps.

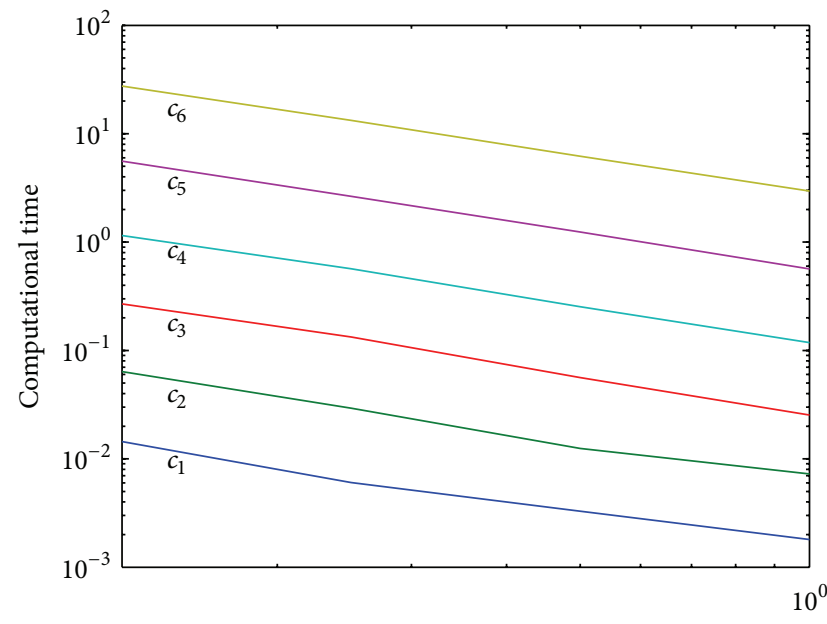

$\Delta t$

(a)

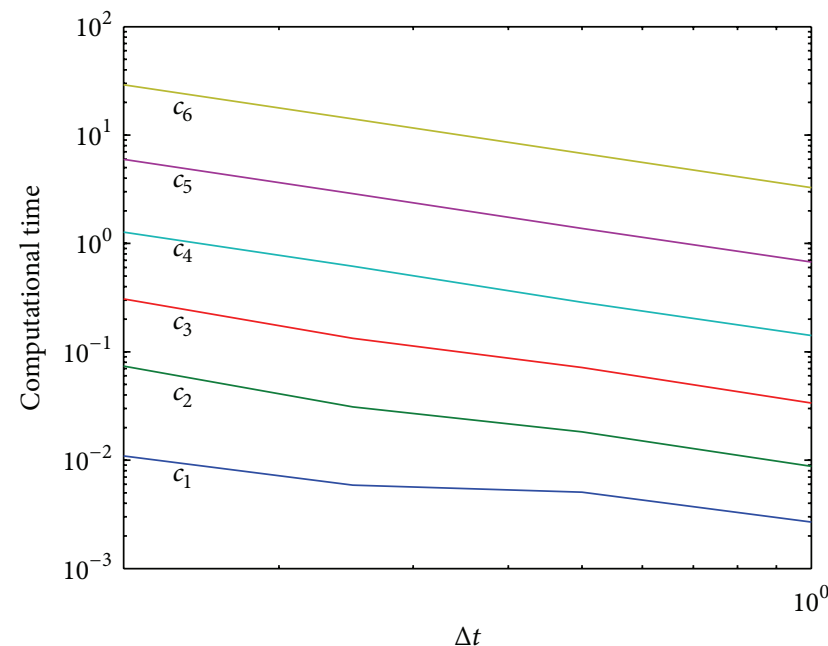

(b)

FIGURE 8: The computational time of the one-sided and two-sided splitting schemes: one-sided splitting over $A$ (a) and two-sided splitting scheme alternating between $A$ and $B$ (b) with $1, \ldots, 6$ iterative steps.

The reaction and scattering matrices are

$$
\begin{aligned}
\Lambda_{1}= & \left(\begin{array}{ccccc}
\lambda_{1} & 0 & & & \\
0 & \lambda_{1} & 0 & & \\
& \ddots & \ddots & \ddots & \\
& & 0 & \lambda_{1} & 0 \\
& & & 0 & \lambda_{1}
\end{array}\right) \in \mathbb{R}^{m \times m}, \\
\Lambda_{2}= & \left(\begin{array}{ccccc}
\lambda_{2} & 0 & & & \\
0 & \lambda_{2} & 0 & & \\
& \ddots & \ddots & \ddots & \\
& & 0 & \lambda_{2} & 0 \\
& & & 0 & \lambda_{2}
\end{array}\right) \in \mathbb{R}^{m \times m},
\end{aligned}
$$

where $m$ is the number of spatial points.
In the first algorithm, we use the following approximation of the integral term:

$$
\int_{0}^{t} \lambda_{2} c(x, t) d t \approx \lambda_{2} t c(x, t) .
$$

We use the following recurrence relations, distinguishing between even and odd iterations.

For the odd iterations, $i=2 \widetilde{m}+1$, with $\widetilde{m}=0,1,2, \ldots$, we have

$$
\begin{aligned}
\mathbf{C}_{i}(t)= & \exp \left(A\left(t-t^{n}\right)\right) \mathbf{C}\left(t^{n}\right) \\
& +\int_{t^{n}}^{t} \exp ((t-s) A) B(s) \mathbf{C}_{i-1}(s) d s,
\end{aligned}
$$




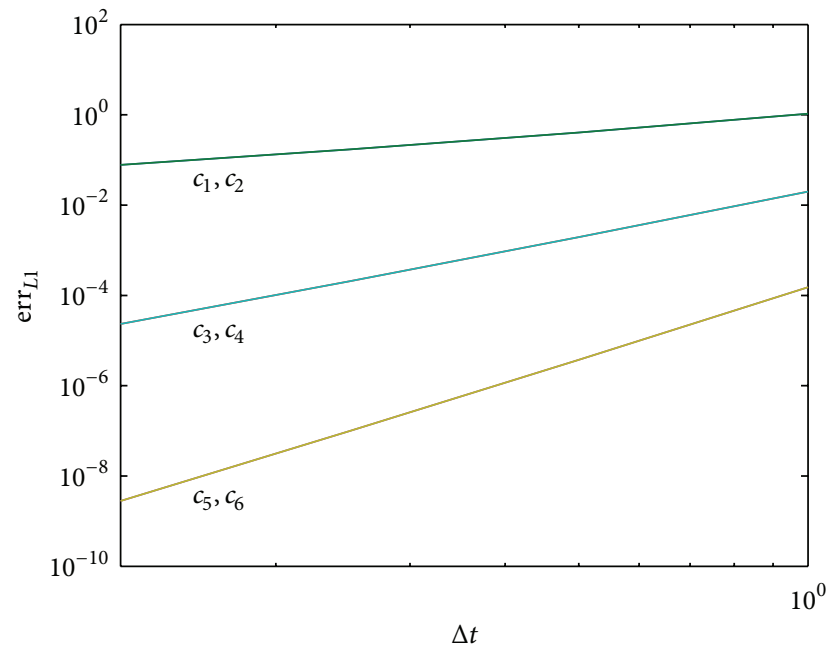

(a)

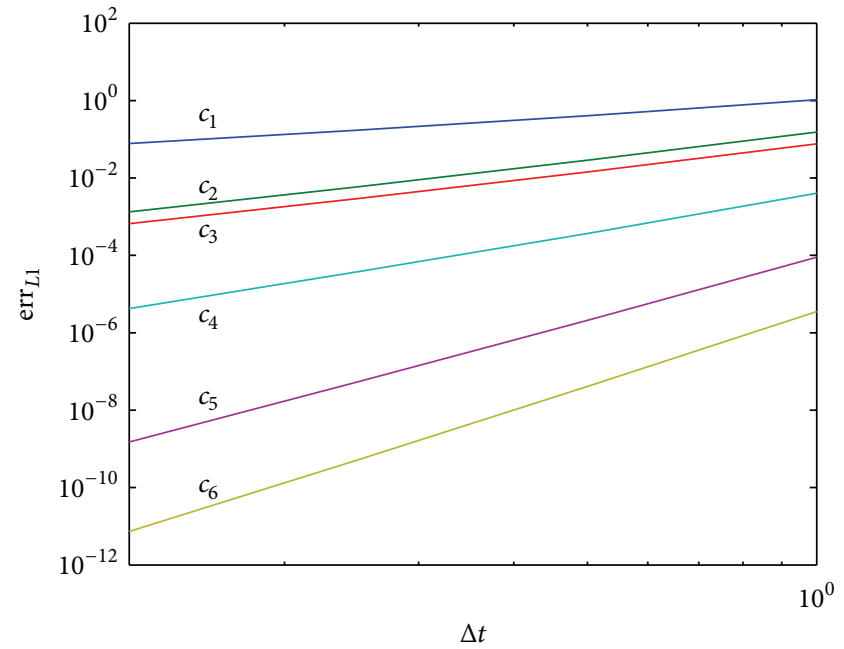

(b)

Figure 9: Numerical errors of the one-sided and two-sided splitting schemes for an optimised approach: one-sided splitting over $A$ (a) and two-sided splitting scheme alternating between $A$ and $B(\mathrm{~b})$ with $1, \ldots, 6$ iterative steps.

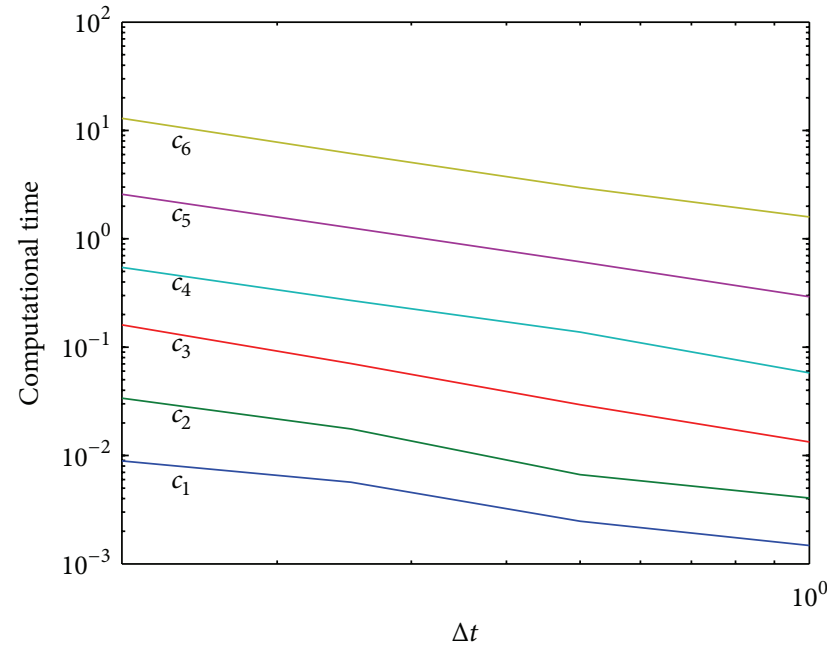

(a)

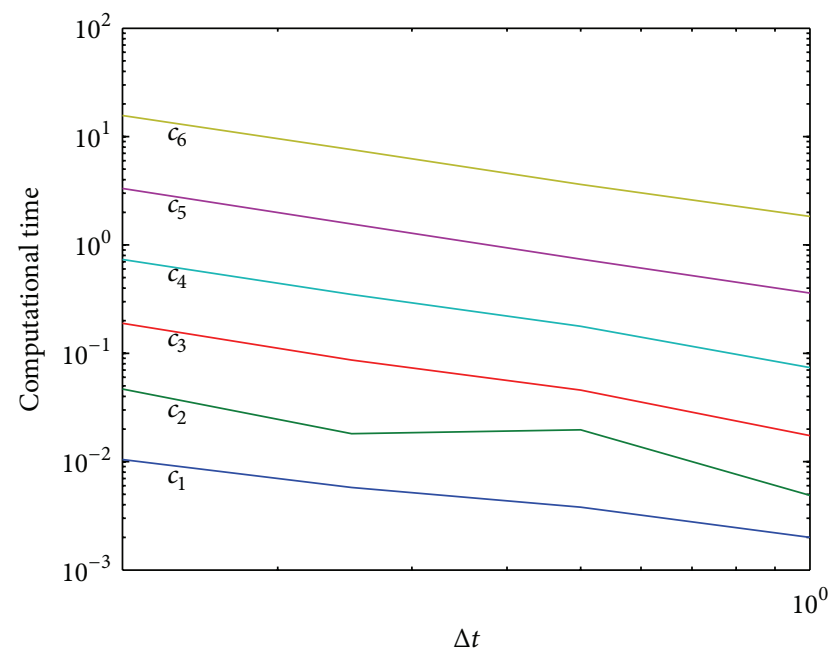

(b)

FIGURE 10: The computational time of the one-sided and two-sided splitting schemes for an optimised approach: one-sided splitting over $A$ (a) and two-sided splitting scheme alternating between $A$ and $B$ (b) with $1, \ldots, 6$ iterative steps.

For the even iterations, $i=2 \widetilde{m}$, with $\widetilde{m}=0,1,2, \ldots$, we have

$$
\begin{array}{r}
\mathbf{C}_{i}(t)=\exp \left(\int_{0}^{t-t^{n}} B(\widetilde{s}) d \widetilde{s}\right) \mathbf{C}\left(t^{n}\right) \\
+\int_{t^{n}}^{t} \exp \left(\int_{0}^{t-s} B(\widetilde{s}) d \widetilde{s}\right) A \mathbf{C}_{i-1}(s) d s, \\
t \in\left(t^{n}, t^{n+1}\right],
\end{array}
$$

where $\int_{0}^{t-t^{n}} B(\widetilde{s}) d \widetilde{s}=\Lambda_{1}\left(t-t^{n}\right)+\Lambda_{2}\left(\left(t-t^{n}\right)^{2} / 2\right)$.

For the reference solution, we apply a fine time-spatial scale without decoupling the equations.
For the algorithms, we employ novel Algorithm 5 and we obtain $\widetilde{A}=A-\Lambda_{1}$ and $\widetilde{B}=\Lambda_{2}$.

The matrices for the novel algorithm are

$$
\begin{gathered}
\widetilde{\widetilde{A}}=\frac{\widetilde{A}}{2}, \\
\widetilde{\widetilde{B}}=\frac{1}{2} \sqrt{\frac{\widetilde{A} \widetilde{A}^{t}}{4}+\widetilde{B} .}
\end{gathered}
$$

Figure 7 presents the one-sided and two-sided iterative results.

The computational results are given in Figure 8, which presents the one-sided and two-sided iterative results. 
TABLE 4: The computational time of all three experiments.

\begin{tabular}{lc}
\hline Algorithm & Computational time in sec \\
\hline numerical approach-twoSide & 23.8826 \\
numerical approach-oneSideA & 25.4946 \\
numerical approach-oneSideB & 24.1386 \\
\hline exact approach-twoSide & 67.1759 \\
exact approach-oneSideA & 62.7140 \\
exact approach-oneSideB & 70.8573 \\
\hline exact optimised approach-twoSide & 36.4432 \\
exact optimised approach-oneSideA & 29.7267 \\
exact optimised approach-oneSideB & 38.1619 \\
\hline
\end{tabular}

Figure 9 presents the one-sided and two-sided iterative results for an optimised approach.

The computational results are given in Figure 10, which presents the one-sided and two-sided iterative results for an optimised approach.

Table 4 presents the computational times of the three experiments.

Remark 13. For all iterative schemes, we can reach faster results using the optimised approach. Such iterative schemes with fast computations of the exponential matrices accelerate standard splitting schemes. With four or five iterative steps we obtain more accurate results than we did using the expensive standard schemes. One-sided iterative schemes yield the best convergence results.

\section{Conclusions and Discussion}

We presented a coupled model for a transport and kinetic model for deposition species in a plasma environment. We proposed numerical schemes to decompose the flow field and the reaction-collision term. A generalised iterative scheme was discussed and their analysis and benefits were presented.

Such combined splitting schemes help to reduce the computational time required for delicate plasma problems. In the future, we will deal with nonlinear schemes of collision problems in plasma reactors.

\section{Conflict of Interests}

The author declares that there is no conflict of interests regarding the publication of this paper.

\section{References}

[1] J. Geiser, "An iterative splitting approach for linear integrodifferential equations," Applied Mathematics Letters, vol. 26, no. 11, pp. 1048-1052, 2013.

[2] J. Geiser, "Multiscale splitting method for the BoltzmannPoisson equation: application to the dynamics of electrons," International Journal of Differential Equations, vol. 2014, Article ID 178625, 8 pages, 2014.

[3] S. Blanes, F. Casas, and A. Murua, "Error analysis of splitting methods for the time dependent Schrödinger equation," SIAM
Journal on Scientific Computing, vol. 33, no. 4, pp. 1525-1548, 2011.

[4] R. Bhatia, Matrix Analysis, vol. 169 of Graduate Texts in Mathematics, Springer, 1997.

[5] G. Strang, "On the construction and comparison of difference schemes," SIAM Journal on Numerical Analysis, vol. 5, pp. 506517, 1968.

[6] J. Verwer and B. Sportisse, "A note on operator splitting in a stiff linear case," MAS Report R9830, 1998.

[7] Z. Zlatev, Computer Treatment of Large Air Pollution Models, Kluwer Academic, New York, NY, USA, 1995.

[8] J. F. Kanney, C. T. Miller, and C. T. Kelley, "Convergence of iterative split-operator approaches for approximating nonlinear reactive problems," Advances in Water Resources, vol. 26, no. 3, pp. 247-261, 2003.

[9] E. J. Dean, R. Glowinski, and J. L. Lions, "An operator splitting approach to multilevel methods," Applied Mathematics Letters, vol. 15, no. 4, pp. 505-511, 2002.

[10] J. Geiser, Iterative Splitting Methods for Differential Equations, CRC Press, Chapman \& Hall, 2011.

[11] J. Geiser, "Computing exponential for iterative splitting methods: algorithms and applications," Journal of Applied Mathematics, vol. 2011, Article ID 193781, 27 pages, 2011.

[12] S. H. Cheng, N. J. Higham, C. S. Kenney, and A. J. Laub, "Approximating the logarithm of a matrix to specified accuracy," SIAM Journal on Matrix Analysis \& Applications, vol. 22, no. 4, pp. 1112-1125, 2001.

[13] E. D. Denman and J. Beavers, "The matrix sign function and computations in systems," Applied Mathematics and Computation, vol. 2, no. 1, pp. 63-94, 1976. 


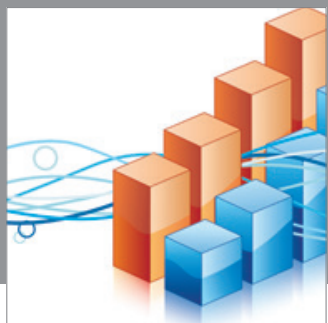

Advances in

Operations Research

mansans

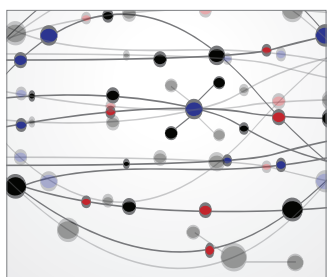

The Scientific World Journal
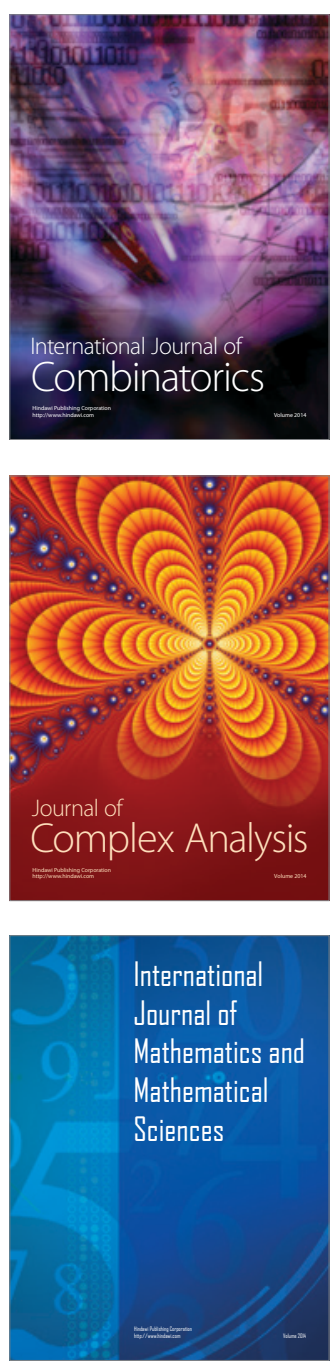
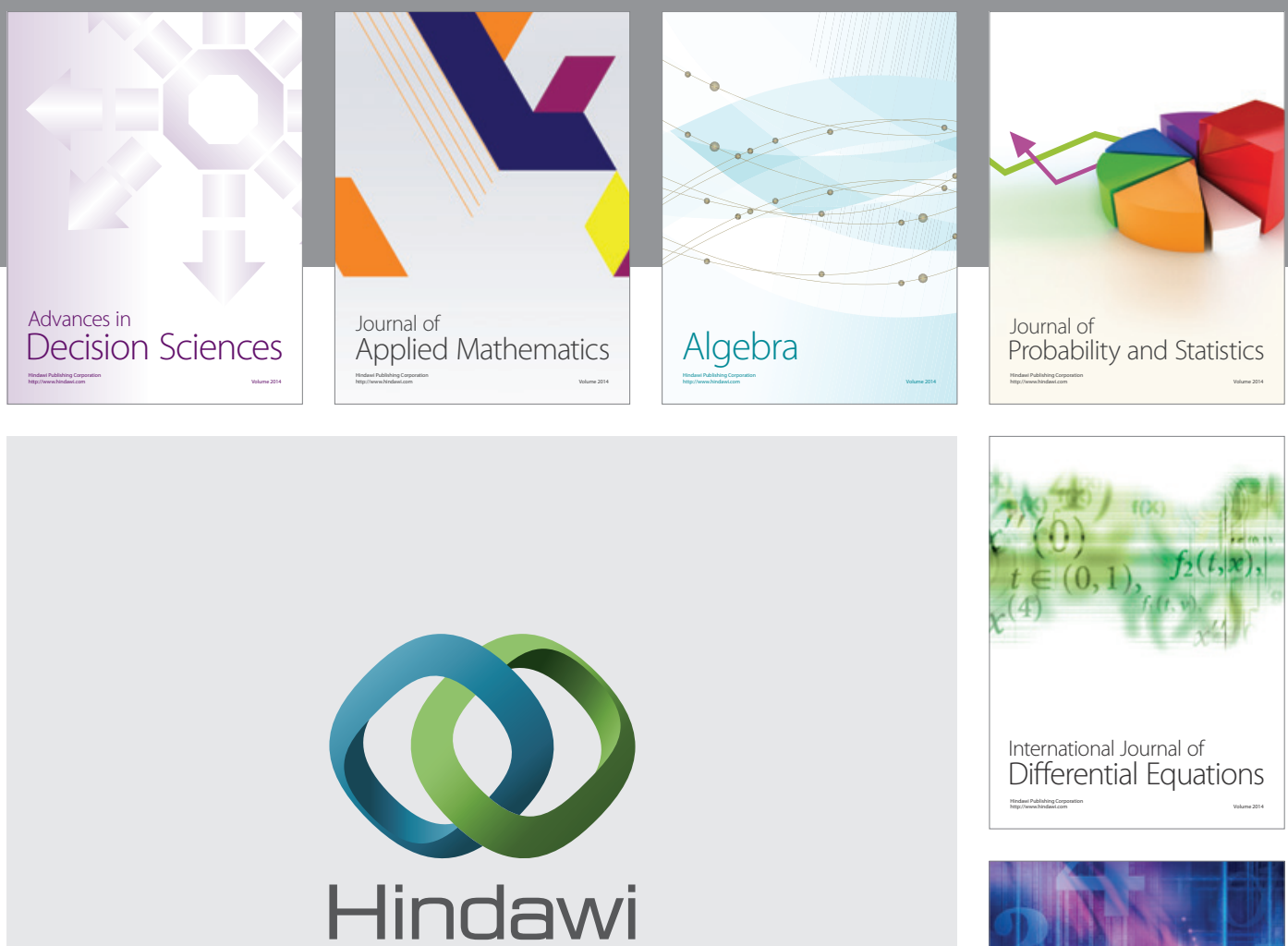

Submit your manuscripts at http://www.hindawi.com
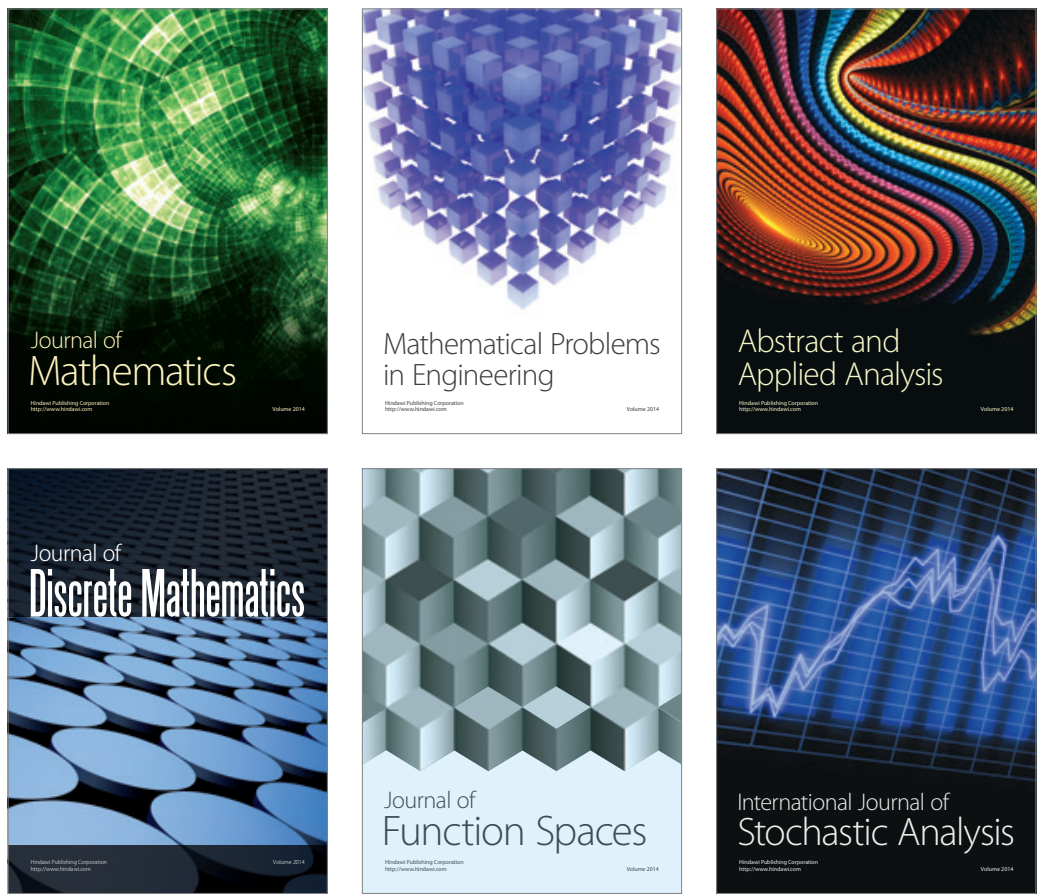

Journal of

Function Spaces

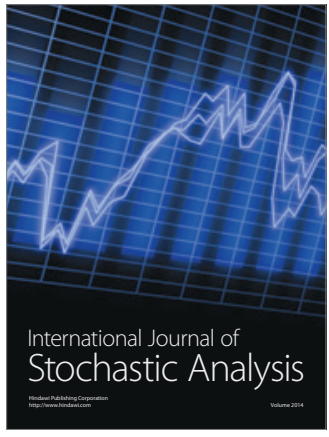

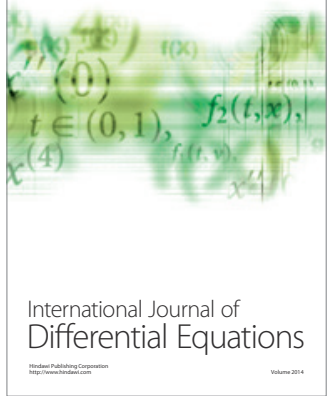
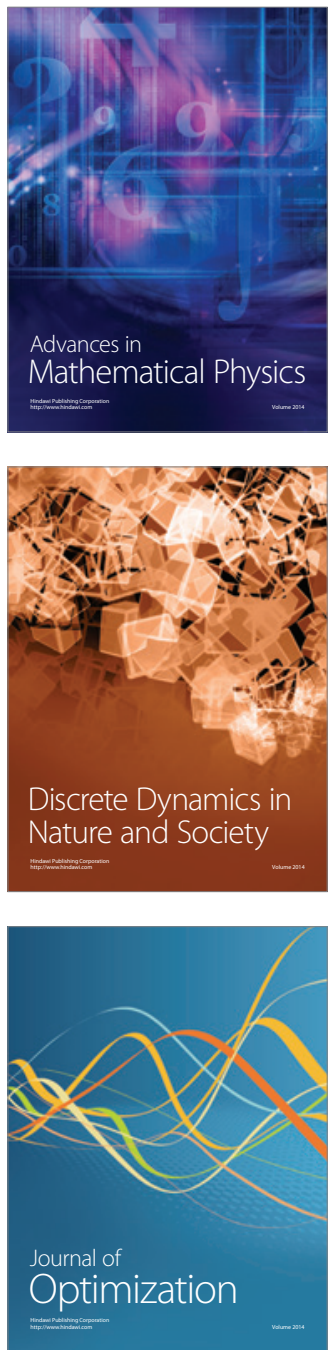